Journal of Applied AnAlysis

Vol. 12, No. 1 (2006), pp. 19-36

\title{
AN ELASTIC CONTACT PROBLEM WITH ADHESION AND NORMAL COMPLIANCE
}

\author{
M. SOFONEA and A. MATEI \\ Received October 6, 2003 and, in revised form, January 24, 2005
}

\begin{abstract}
We study a mathematical problem describing the frictionless adhesive contact between an elastic body and a foundation. The adhesion process is modelled by a surface variable, the bonding field, and the contact is modelled with a normal compliance condition; the tangential shear due to the bonding field is included; the elastic constitutive law is assumed to be nonlinear and the process is quasistatic. The problem is formulated as a nonlinear system in which the unknowns are the displacement, the stress and the bonding field. The existence of a unique weak solution for the problem is established by using arguments for differential equations followed by the construction of an appropriate contraction mapping.
\end{abstract}

\section{Introduction}

The adhesive contact between bodies, when a glue is added to prevent relative motion of the surfaces, has received recently increased attention in the mathematical literature. Basic modelling can be found in $[5,7,8]$. Analysis of models for frictionless adhesive contact can be found in

2000 Mathematics Subject Classification. 74M15, 74F99, 74G25.

Key words and phrases. Quasistatic contact, normal compliance, adhesion, elastic material, weak solution, fixed point.

ISSN 1425-6908（C) Heldermann Verlag. 
$[1,2,3,4,6,9,11,12,18]$. The models studied in $[1,2,9]$ and [18] involve special geometries or settings; indeed, in [9] the problem of a beam in adhesive contact can be found, in $[1,2]$ the adhesive contact of a membrane was investigated in the quasistatic and dynamic case, respectively, and in [18] a model of antiplane adhesive contact for linearly elastic materials problem was shown to have a weak solution. Moreover, a novel application of the theory is in the medical field of prosthetic limbs where the bonding between the bone-implant and the tissue is of considerable importance, since debonding may lead to decrease in the persons ability to use the artificial limb or joint (see $[15,16]$ and references therein).

The novelty in these papers is the introduction of a surface internal variable, the bonding field, which describes the pointwise fractional density of active bonds on the contact surface, and sometimes referred to as the 'intensity of adhesion'. We refer the reader to the extensive bibliography on the subject in $[14,15,17]$.

In this paper we continue the investigation of adhesive problems begun in $[3,4,6,11,12]$. There, models for dynamic or quasistatic process of frictionless adhesive contact between a deformable body and a foundation have been analyzed and simulated; the contact was described with normal compliance or was assumed to be bilateral, and the behavior of the material was modeled with a nonlinear Kelvin-Voigt viscoelastic constitutive law; the models included the bonding field as an additional dependent variable, defined and evolving on the contact surface. The existence of a unique weak solution to the models have been obtained by using arguments of evolutionary equations in Banach spaces and fixed-point theorems.

In this paper we deal with the quasistatic process of frictionless adhesive contact, using the bonding field and the normal compliance contact condition, too. However, we consider the case of nonlinear elastic materials, which consists the trait of novelty of the present paper. We derive a variational formulation of the model and prove the existence a unique weak solution to the model. To this end we use similar arguments as in $[3,4,6,11,12]$ but with a different choice of functionals and operators, since the physical setting here and in the above mentioned papers is different. Moreover, the existence result we obtain in this paper is valid only when the sizes of some constants are restricted, which quite often is a feature in contact problems involving inviscid materials. Indeed, it was found in [11] that even vanishing amounts of viscosity allow to prove the well-posedness of the model, without any smallness assumptions on the constitutive contact functions.

Our paper is structured as follows. In Section 2 we present some notation and preliminary material. In Section 3 we state the mechanical model of elastic frictionless contact with adhesion and normal compliance. In Section 4 we list the assumptions on the data, derive the variational formulation to 
the problem and state our main existence and uniqueness result, Theorem 4.1. The proof of this theorem is given in Section 5; it is carried out in several steps and is based on arguments for differential equations and a fixed point theorem.

\section{Notations and preliminaries}

Everywhere in this paper we denote by $\mathbb{S}$ the space of second order symmetric tensors on $\mathbb{R}^{d}(d=1,2,3)$; "." and $|\cdot|$ represent the inner product and the Euclidean norm on $\mathbb{R}^{d}$ and $\mathbb{S}$, respectively. Thus, for every $\boldsymbol{u}, \boldsymbol{v} \in \mathbb{R}^{d}, \boldsymbol{u} \cdot \boldsymbol{v}=u_{i} v_{i},|\boldsymbol{v}|=(\boldsymbol{v} \cdot \boldsymbol{v})^{1 / 2}$, and for every $\boldsymbol{\sigma}, \boldsymbol{\tau} \in \mathbb{S}$, $\boldsymbol{\sigma} \cdot \boldsymbol{\tau}=\sigma_{i j} \tau_{i j},|\boldsymbol{\tau}|=(\boldsymbol{\tau} \cdot \boldsymbol{\tau})^{1 / 2}$. Here and below, the indices $i, j, k, h$ run between 1 and $d$ and the summation convention over repeated indices is adopted.

Let $\Omega \subset \mathbb{R}^{d}$ be a bounded domain with a Lipschitz boundary $\Gamma$. In what follows we use the standard notation for the Lebesgue and Sobolev spaces associated to $\Omega$ and $\Gamma$, and the index that follows a comma indicates a derivative with respect to the corresponding component of the spatial variable $\boldsymbol{x} \in \Omega$. We shall also use the notation

$$
\begin{array}{ll}
H=L^{2}(\Omega)^{d}=\left\{\boldsymbol{u}=\left(u_{i}\right) \mid u_{i} \in L^{2}(\Omega)\right\}, & H_{1}=\left\{\boldsymbol{u}=\left(u_{i}\right) \mid u_{i} \in H^{1}(\Omega)\right\}, \\
\mathcal{H}=\left\{\boldsymbol{\sigma}=\left(\sigma_{i j}\right) \mid \sigma_{i j}=\sigma_{j i} \in L^{2}(\Omega)\right\}, & \mathcal{H}_{1}=\left\{\boldsymbol{\sigma} \in \mathcal{H} \mid \sigma_{i j, j} \in L^{2}(\Omega)\right\} .
\end{array}
$$

The spaces $H, \mathcal{H}, H_{1}$ and $\mathcal{H}_{1}$ are real Hilbert spaces endowed with the inner products given by

$$
\begin{aligned}
(\boldsymbol{u}, \boldsymbol{v})_{H} & =\int_{\Omega} u_{i} v_{i} d x, & (\boldsymbol{\sigma}, \boldsymbol{\tau})_{\mathcal{H}} & =\int_{\Omega} \sigma_{i j} \tau_{i j} d x, \\
(\boldsymbol{u}, \boldsymbol{v})_{H_{1}} & =(\boldsymbol{u}, \boldsymbol{v})_{H}+(\boldsymbol{\varepsilon}(\boldsymbol{u}), \boldsymbol{\varepsilon}(\boldsymbol{v}))_{\mathcal{H}}, & (\boldsymbol{\sigma}, \boldsymbol{\tau})_{\mathcal{H}_{1}} & =(\boldsymbol{\sigma}, \boldsymbol{\tau})_{\mathcal{H}}+(\operatorname{Div} \boldsymbol{\sigma}, \operatorname{Div} \boldsymbol{\tau})_{H},
\end{aligned}
$$

respectively. Here $\varepsilon: H_{1} \rightarrow \mathcal{H}$ and Div $: \mathcal{H}_{1} \rightarrow H$ are the deformation and the divergence operators, respectively, defined by

$$
\boldsymbol{\varepsilon}(\boldsymbol{u})=\left(\varepsilon_{i j}(\boldsymbol{u})\right), \quad \varepsilon_{i j}(\boldsymbol{u})=\frac{1}{2}\left(u_{i, j}+u_{j, i}\right), \quad \operatorname{Div} \boldsymbol{\sigma}=\left(\sigma_{i j, j}\right) .
$$

The associated norms on the spaces $H, \mathcal{H}, H_{1}$ and $\mathcal{H}_{1}$ are denoted by $\|\cdot\|_{H}$, $\|\cdot\|_{\mathcal{H}},\|\cdot\|_{H_{1}}$ and $\|\cdot\|_{\mathcal{H}_{1}}$, respectively.

Since the boundary $\Gamma$ is Lipschitz continuous, the unit outward normal vector $\boldsymbol{\nu}$ on the boundary is defined a.e. For every vector field $\boldsymbol{v} \in H_{1}$ we use the notation $\boldsymbol{v}$ for the trace of $\boldsymbol{v}$ on $\Gamma$ and we denote by $v_{\nu}$ and $\boldsymbol{v}_{\tau}$ the normal and the tangential components of $\boldsymbol{v}$ on the boundary, given by

$$
v_{\nu}=\boldsymbol{v} \cdot \boldsymbol{\nu}, \quad \boldsymbol{v}_{\tau}=\boldsymbol{v}-v_{\nu} \boldsymbol{\nu}
$$


For a regular (say $C^{1}$ ) stress field $\sigma$, the application of its trace on the boundary to $\boldsymbol{\nu}$ is the Cauchy stress vector $\boldsymbol{\sigma} \boldsymbol{\nu}$. We define, similarly, the normal and tangential components of the stress on the boundary by the formulas

$$
\sigma_{\nu}=(\boldsymbol{\sigma} \boldsymbol{\nu}) \cdot \boldsymbol{\nu}, \quad \boldsymbol{\sigma}_{\tau}=\boldsymbol{\sigma} \boldsymbol{\nu}-\sigma_{\nu} \boldsymbol{\nu}
$$

and we recall that the following Green's formula holds:

$$
(\boldsymbol{\sigma}, \boldsymbol{\varepsilon}(\boldsymbol{v}))_{\mathcal{H}}+(\operatorname{Div} \boldsymbol{\sigma}, \boldsymbol{v})_{H}=\int_{\Gamma} \boldsymbol{\sigma} \boldsymbol{\nu} \cdot \boldsymbol{v} d a \quad \forall \boldsymbol{v} \in H_{1}
$$

Finally, for every real Banach space $X$ we use the classical notation for the spaces $L^{p}(0, T ; X)$ and $W^{m, p}(0, T ; X), 1 \leq p \leq+\infty, m=1,2, \ldots$, and we use the dot above to indicate the derivative with respect to the time variable. Moreover, for a real number $r$, we use $r_{+}$to represent its positive part, that is $r_{+}=\max \{0, r\}$.

\section{Problem statement}

We consider an elastic body which occupies the bounded domain $\Omega \subset \mathbb{R}^{d}$ with a Lipschitz continuous outer surface $\Gamma$ that is divided into three disjoint measurable parts $\Gamma_{1}, \Gamma_{2}$ and $\Gamma_{3}$ such that meas $\Gamma_{1}>0$. Let $T>0$ and let $[0, T]$ denote the time interval of interest. The body is clamped on $\Gamma_{1} \times(0, T)$ and, therefore, the displacement field vanishes there. A volume force of density $\boldsymbol{f}_{0}$ acts in $\Omega \times(0, T)$ and surface tractions of density $\boldsymbol{f}_{2}$ act on $\Gamma_{2} \times(0, T)$. The body is in adhesive contact with an obstacle, the so called foundation, over the potential contact surface $\Gamma_{3}$.

We denote by $\boldsymbol{u}$ the displacement field, by $\boldsymbol{\sigma}$ the stress tensor field and by $\varepsilon(\boldsymbol{u})$ the linearized strain tensor. We assume that the material is elastic and obeys the constitutive law

$$
\boldsymbol{\sigma}=\mathcal{E}(\boldsymbol{\varepsilon}(\boldsymbol{u})) \quad \text { in } \Omega \times(0, T),
$$

where $\mathcal{E}$ is a given nonlinear constitutive function which will be described below. To simplify the notation, here and below we do not indicate explicitly the dependence of various functions on the variables $\boldsymbol{x} \in \Omega \cup \Gamma$ and $t \in[0, T]$.

As examples of nonlinear constitutive functions $\mathcal{E}$ we may consider

$$
\mathcal{E}(\varepsilon)=\mathcal{A} \varepsilon+\beta\left(\varepsilon-\mathcal{P}_{K} \varepsilon\right)
$$

in which $\mathcal{A}$ is a fourth-order tensor, $\beta>0, K$ is a closed convex subset of $\mathbb{S}$ such that $\mathbf{0} \in K$ and $\mathcal{P}_{K}: \mathbb{S} \rightarrow \mathbb{K}$ denotes the projection map. A second example is provided by a nonlinear Hencky material when

$$
\mathcal{E}(\varepsilon)=\kappa(\operatorname{tr} \varepsilon) \boldsymbol{I}_{d}+\psi\left(\left|\varepsilon^{D}\right|^{2}\right) \varepsilon^{D} .
$$


Here, $\kappa>0$ is a material coefficient, $\boldsymbol{I}_{d}$ is the identity tensor of the second order, $\psi: \mathbb{R} \rightarrow \mathbb{R}$ is a constitutive function and $\varepsilon^{D}=\varepsilon^{D}(\boldsymbol{u})$ denotes the deviatoric part of $\varepsilon=\varepsilon(\boldsymbol{u})$ that is

$$
\varepsilon^{D}=\varepsilon-\frac{1}{d}(\operatorname{tr} \varepsilon) \boldsymbol{I}_{d}
$$

We describe now the conditions on the contact surface $\Gamma_{3}$.

Following $[7,8]$, we introduce the surface state variable $\beta$, called the bonding field, which is a measure of the fractional intensity of adhesion between the surface and the foundation. This variable is restricted to values between $\beta=0$, when there are no active bonds, and $\beta=1$ when all the bonds are active. When $0<\beta<1$ it measures the fraction of active bonds, that is partial adhesion takes place.

We assume that the normal stress satisfies the condition of normal compliance with adhesion,

$$
-\sigma_{\nu}=p_{\nu}\left(u_{\nu}\right)-\gamma_{\nu} \beta^{2}\left(-R_{L}\left(u_{\nu}\right)\right)_{+} \quad \text { on } \Gamma_{3} \times(0, T) .
$$

Here $p_{\nu}$ is a prescribed and nonnegative function such that $p_{\nu}(r)=0$ for $r \leq$ 0 and the normal displacement $u_{\nu}$, when positive, represents the penetration of the body into the foundation. The second term is the contribution of the bonding to the surface tension in which $\gamma_{\nu}$ is the bonding stiffness constant, $L>0$ is a characteristic lenght of the bonds (see, e.g. [14]) and $R_{L}: \mathbb{R} \rightarrow \mathbb{R}$ is the truncation function defined by

$$
R_{L}(s)= \begin{cases}-L & \text { if } \quad s \leq-L \\ s & \text { if } \quad|s|<L \\ L & \text { if } \quad s \geq L\end{cases}
$$

The introduction of $R_{L}$ is motivated by the observation that if the extension is more than $L$, the glue extends plastically without offering additional tensile traction. However, by choosing $L$ sufficiently large, say larger than the size of the system, we recover the case where the traction is linear in the extension. Thus, the contribution of the adhesive to the normal traction is represented by $\gamma_{\nu} \beta^{2}\left(-R_{L}\left(u_{\nu}\right)\right)_{+}$; the adhesive traction is tensile, and is proportional, with proportionality coefficient $\gamma_{\nu}$, to the square of the intensity of adhesion, and to the normal displacement, but as long as it does not exceed the bond length $L$. The maximal tensile traction is $\gamma_{\nu} L$.

As examples of normal compliance functions we may consider

$$
p_{\nu}(r)=c r_{+}
$$

or

$$
p_{\nu}(r)= \begin{cases}c r_{+} & \text {if } r \leq \alpha \\ c \alpha & \text { if } r>\alpha,\end{cases}
$$


where $c>0$ and $\alpha>0$ are surface parameters. Using (3.6) in (3.4) it follows that $u_{\nu}>0 \Rightarrow-\sigma_{\nu}=c u_{\nu}$, that is the reaction of the obstacle is proportional to the penetration. Using (3.7) in (3.4) it follows that $u_{\nu}>$ $\alpha \Rightarrow-\sigma_{\nu}=c \alpha$ which shows that if the penetration exceeds the value $\alpha$ then the obstacle offers no additional resistance to penetration.

As in $[4,11]$ we assume that the resistance to tangential motion is generated mainly by the glue, and the frictional traction can be neglected. In particular, when all the adhesive bonds are inactive, or broken, the motion is frictionless. A different assumption, taking friction into account, can be found in $[14,15,16]$. Thus, the tangential traction depends only on the bonding field and on the tangential displacement, that is

$$
-\boldsymbol{\sigma}_{\tau}=\boldsymbol{p}_{\tau}\left(\beta, \boldsymbol{u}_{\tau}\right) \quad \text { on } \Gamma_{3} \times(0, T) .
$$

In particular, we can consider the case

$$
\boldsymbol{p}_{\tau}(\beta, \boldsymbol{r})=\left\{\begin{array}{lll}
q_{\tau}(\beta) \boldsymbol{r} & \text { if } & |\boldsymbol{r}| \leq L \\
q_{\tau}(\beta) \frac{L}{|\boldsymbol{r}|} \boldsymbol{r} & \text { if } & |\boldsymbol{r}|>L
\end{array}\right.
$$

where $L>0$ is the characteristic length of the bond and $q_{\tau}$ is a nonnegative tangential stiffness function. Note that in this case the tangential traction is opposite to the tangential displacement.

As in $[4,11]$ we assume that the evolution of the bonding field is governed by the differential equation

$$
\dot{\beta}=H_{a d}\left(\beta, R_{L}(|\boldsymbol{u}|)\right) \quad \text { on } \quad \Gamma_{3} \times(0, T),
$$

where $H_{a d}$ is a general function discussed below, which may change sign. This condition allows for rebonding after debonding took place and it allows for possible cycles of debonding and rebonding. As examples of such functions consider

$$
H_{a d}(\beta, r)=-\gamma_{0} \beta_{+} r^{2}
$$

or

$$
H_{a d}(\beta, r)=-\gamma_{0} \frac{\beta_{+}}{1+\beta_{+}} r^{2},
$$

where $\gamma_{0}>0$ is a bonding coefficient.

Let $\beta_{0}$ be the initial bonding field. We assume that the process is quasistatic and therefore, using (3.1), (3.4), (3.8) and (3.10), we obtain the following mechanical model for the elastic frictionless contact problem with adhesion and normal compliance. 
Problem $P$. Find a displacement field $\boldsymbol{u}: \Omega \times[0, T] \rightarrow \mathbb{R}^{d}$, a stress field $\boldsymbol{\sigma}: \Omega \times[0, T] \rightarrow \mathbb{S}$ and a bonding field $\beta: \Gamma_{3} \times[0, T] \rightarrow[0,1]$ such that

$$
\begin{aligned}
\boldsymbol{\sigma} & =\mathcal{E}(\varepsilon(\boldsymbol{u})) & & \text { in } \Omega \times(0, T), \\
\operatorname{Div} \boldsymbol{\sigma}+\boldsymbol{f}_{0} & =\mathbf{0} & & \text { in } \Omega \times(0, T), \\
\boldsymbol{u} & =\mathbf{0} & & \text { on } \Gamma_{1} \times(0, T), \\
\boldsymbol{\sigma} \boldsymbol{\nu} & =\boldsymbol{f}_{2} & & \text { on } \Gamma_{2} \times(0, T), \\
-\sigma_{\nu} & =p_{\nu}\left(u_{\nu}\right)-\gamma_{\nu} \beta^{2}\left(-R_{L}\left(u_{\nu}\right)\right)+ & & \text { on } \Gamma_{3} \times(0, T), \\
-\boldsymbol{\sigma}_{\tau} & =\boldsymbol{p}_{\tau}\left(\beta, \boldsymbol{u}_{\tau}\right) & & \text { on } \Gamma_{3} \times(0, T), \\
\dot{\beta} & =H_{a d}\left(\beta, R_{L}(|\boldsymbol{u}|)\right) & & \text { on } \Gamma_{3} \times(0, T), \\
\beta(0) & =\beta_{0} & & \text { on } \Gamma_{3} .
\end{aligned}
$$

Note that here (3.14) are the equilibrium equations, (3.15) and (3.16) are the displacement and traction boundary conditions, respectively, and (3.20) represent the initial condition for the bonding field.

\section{Variational formulation}

We turn now to the variational formulation of Problem $P$. To this end we denote by $V$ the closed subspace of $H_{1}$ defined by

$$
V=\left\{\boldsymbol{v} \in H_{1} \mid \boldsymbol{v}=\mathbf{0} \text { on } \Gamma_{1}\right\} .
$$

Since meas $\Gamma_{1}>0$, Korn's inequality holds and there exists a constant $C_{K}>0$, that depends only on $\Omega$ and $\Gamma_{1}$, such that $\|\varepsilon(\boldsymbol{v})\|_{\mathcal{H}} \geq C_{K}\|\boldsymbol{v}\|_{H_{1}}$ for all $\boldsymbol{v} \in V$. A proof of Korn's inequality may be found in [13, p. 79]. On $V$ we consider the inner product and the associated norm given by

$$
(\boldsymbol{u}, \boldsymbol{v})_{V}=(\varepsilon(\boldsymbol{u}), \varepsilon(\boldsymbol{v}))_{\mathcal{H}}, \quad\|\boldsymbol{v}\|_{V}=\|\varepsilon(\boldsymbol{v})\|_{\mathcal{H}} \quad \forall \boldsymbol{u}, \boldsymbol{v} \in V .
$$

It follows from Korn's inequality that $\|\cdot\|_{H_{1}}$ and $\|\cdot\|_{V}$ are equivalent norms on $V$ and therefore $\left(V,\|\cdot\|_{V}\right)$ is a real Hilbert space. Moreover, by the Sobolev trace theorem, there exists a constant $c_{0}$, depending only on $\Omega, \Gamma_{1}$ and $\Gamma_{3}$, such that

$$
\|\boldsymbol{v}\|_{L^{2}\left(\Gamma_{3}\right)^{d}} \leq c_{0}\|\boldsymbol{v}\|_{V} \quad \forall \boldsymbol{v} \in V
$$

In the study of the mechanical problem (3.13)-(3.20), we assume that the elasticity operator satisfies: 


$$
\left\{\begin{aligned}
& \text { (a) } \quad \mathcal{E}: \Omega \times \mathbb{S} \rightarrow \mathbb{S} ; \\
& \text { (b) } \quad \text { There exists } L_{\mathcal{E}}>0 \text { such that } \\
&\left|\mathcal{E}\left(\boldsymbol{x}, \varepsilon_{2}\right)-\mathcal{E}\left(\boldsymbol{x}, \varepsilon_{2}\right)\right| \leq L_{\mathcal{E}}\left|\varepsilon_{1}-\varepsilon_{2}\right| \\
& \forall \varepsilon_{1}, \varepsilon_{2} \in \mathbb{S}, \text { a.e. } \boldsymbol{x} \in \Omega ; \\
& \text { (c) } \quad \text { There exists } m_{\mathcal{E}}>0 \text { such that } \\
&\left(\mathcal{E}\left(\boldsymbol{x}, \varepsilon_{1}\right)-\mathcal{E}\left(\boldsymbol{x}, \varepsilon_{2}\right)\right) \cdot\left(\varepsilon_{1}-\varepsilon_{2}\right) \geq m_{\mathcal{E}}\left|\varepsilon_{1}-\varepsilon_{2}\right|^{2} \\
& \forall \varepsilon_{1}, \varepsilon_{2} \in \mathbb{S}, \text { a.e. } \boldsymbol{x} \in \Omega ; \\
& \text { (d) } \quad \text { The mapping } \boldsymbol{x} \mapsto \mathcal{E}(\boldsymbol{x}, \boldsymbol{\varepsilon}) \text { is Lebesgue measurable on } \Omega \\
& \text { for any } \boldsymbol{\varepsilon} \in \mathbb{S} ; \\
& \text { (e) } \quad \text { The mapping } \boldsymbol{x} \mapsto \mathcal{E}(\boldsymbol{x}, \mathbf{0}) \in \mathcal{H}
\end{aligned}\right.
$$

Clearly, a family of elasticity operators satisfying condition (4.2) is provided by the linearly elastic materials

$$
\sigma_{i j}=\mathcal{A}_{i j h k} \varepsilon_{h k}
$$

if the elasticity tensor $\mathcal{A}=\left(\mathcal{A}_{i j h k}\right): \mathbb{S} \rightarrow \mathbb{S}$ is symmetric and positive definite. Also, if $\mathcal{A}$ satisfies the conditions above, by using the properties of the projection map, it can be proved that the operator $\mathcal{E}$ defined by (3.2) satisfies conditions (4.2). Finally, recall that under appropriate assumptions on the constitutive function $\psi$ it can be shown that the operator $\mathcal{E}$ defined in (3.3) satisfies conditions (4.2), see [10, p. 125] for details. We conclude that our results below are valid for the adhesive contact problems associated with these elastic constitutive laws.

The normal compliance function $p_{\nu}$ and the tangential contact function $\boldsymbol{p}_{\tau}$ satisfy:

$$
\begin{cases}\text { (a) } \quad & p_{\nu}: \Gamma_{3} \times \mathbb{R} \rightarrow \mathbb{R}_{+} ; \\ \text {(b) } \quad & \text { There exists } L_{\nu}>0 \text { such that } \\ & \left|p_{\nu}\left(\boldsymbol{x}, r_{1}\right)-p_{\nu}\left(\boldsymbol{x}, r_{2}\right)\right| \leq L_{\nu}\left|r_{1}-r_{2}\right| \\ & \forall r_{1}, r_{2} \in \mathbb{R}, \text { a.e. } \boldsymbol{x} \in \Gamma_{3} ; \\ \text { (c) } \quad \text { The mapping } \boldsymbol{x} \mapsto p_{\nu}(\boldsymbol{x}, r) \text { is Lebesgue measurable } \\ & \text { on } \Gamma_{3}, \text { for any } r \in \mathbb{R} ; \\ \text { (d) } \quad r \leq 0 \Rightarrow p_{\nu}(\boldsymbol{x}, r)=0, \text { a.e. } \boldsymbol{x} \in \Gamma_{3} .\end{cases}
$$




$$
\begin{cases}\text { (a) } & \boldsymbol{p}_{\tau}: \Gamma_{3} \times \mathbb{R} \times \mathbb{R}^{d} \rightarrow \mathbb{R}^{d} ; \\ \text { (b) } & \text { There exists } L_{\tau}>0 \text { such that } \\ & \left|\boldsymbol{p}_{\tau}\left(\boldsymbol{x}, \beta_{1}, \boldsymbol{r}_{1}\right)-\boldsymbol{p}_{\tau}\left(\boldsymbol{x}, \beta_{2}, \boldsymbol{r}_{2}\right)\right| \leq L_{\tau}\left(\left|\beta_{1}-\beta_{2}\right|+\left|\boldsymbol{r}_{1}-\boldsymbol{r}_{2}\right|\right) \\ & \forall \beta_{1}, \beta_{2} \in \mathbb{R}, \boldsymbol{r}_{1}, \boldsymbol{r}_{2} \in \mathbb{R}^{d} \text {, a.e. } \boldsymbol{x} \in \Gamma_{3} ; \\ \text { (c) } & \text { The mapping } \boldsymbol{x} \mapsto \boldsymbol{p}_{\tau}(\boldsymbol{x}, \beta, \boldsymbol{r}) \text { is Lebesgue measurable } \\ & \text { on } \Gamma_{3}, \text { for any } \beta \in \mathbb{R}, \boldsymbol{r} \in \mathbb{R}^{d} ; \\ \text { (d) } & \text { The mapping } \boldsymbol{x} \mapsto \boldsymbol{p}_{\tau}(\boldsymbol{x}, 0,0) \in L^{\infty}\left(\Gamma_{3}\right)^{d} ; \\ \text { (e) } & \boldsymbol{p}_{\tau}(\boldsymbol{x}, \beta, \boldsymbol{r}) \cdot \boldsymbol{\nu}(\boldsymbol{x})=0 \text { for any } \boldsymbol{r} \in \mathbb{R}^{d} \text { such that } \boldsymbol{r} \cdot \boldsymbol{\nu}(\boldsymbol{x})=0, \\ & \text { a.e. } \boldsymbol{x} \in \Gamma_{3} .\end{cases}
$$

Clearly, the normal compliance functions (3.6) and (3.7) satisfy condition (4.3) and, if $q_{\tau}: \mathbb{R} \rightarrow \mathbb{R}$ is a bounded Lipschitz continuous function, then the tangential contact function (3.9) satisfies condition (4.4). We conclude that our results below are valid for the corresponding contact problems.

Next, we assume that the adhesion rate function satisfies:

$$
\begin{cases}\text { (a) } \quad & H_{a d}: \Gamma_{3} \times \mathbb{R} \times \mathbb{R}_{+} \rightarrow \mathbb{R} ; \\ \text { (b) } \quad & \text { There exists } L_{H_{a d}}>0 \text { such that } \\ & \left|H_{a d}\left(\boldsymbol{x}, b_{1}, r_{1}\right)-H_{a d}\left(\boldsymbol{x}, b_{2}, r_{2}\right)\right| \\ & \leq L_{H_{a d}}\left(\left|b_{1}-b_{2}\right|+\left|r_{1}-r_{2}\right|\right) \\ & \forall b_{1}, b_{2} \in \mathbb{R}, \forall r_{1}, r_{2} \in[0, L], \text { a.e. } \boldsymbol{x} \in \Gamma_{3} ; \\ \text { (c) } \quad & \text { The mapping } \boldsymbol{x} \mapsto H_{a d}(\boldsymbol{x}, b, r) \text { is Lebesgue measurable } \\ & \text { on } \Gamma_{3}, \text { for any } b \in \mathbb{R}, r \in[0, L] ; \\ \text { (d) } \quad & \text { The mapping }(b, r) \mapsto H_{a d}(\boldsymbol{x}, b, r) \text { is continuous on } \\ & \mathbb{R} \times[0, L], \text { a.e. } \boldsymbol{x} \in \Gamma_{3} ; \\ \text { (e) } \quad & H_{a d}(\boldsymbol{x}, 0, r)=0 \quad \forall r \in[0, L], \text { a.e. } \boldsymbol{x} \in \Gamma_{3} ; \\ \text { (f) } \quad H_{a d}(\boldsymbol{x}, b, r) \geq 0 \quad \forall b \leq 0, r \in[0, L], \text { a.e. } \boldsymbol{x} \in \Gamma_{3} \quad \text { and } \\ & H_{a d}(\boldsymbol{x}, b, r) \leq 0 \quad \forall b \geq 1, r \in[0, L], \text { a.e. } \boldsymbol{x} \in \Gamma_{3} .\end{cases}
$$

These conditions are chosen to ensure the restriction $0 \leq \beta \leq 1$ for the bonding field, see the proof of Lemma 5.1 below for details. Moreover, it is straightforward to see that if the bonding energy constant $\gamma_{\nu}$ is positive, then the functions $H_{a d}$ in examples (3.11) and (3.12) satisfy (4.5). We 
conclude that all the results below are valid for the models involving the corresponding evolutions of the adhesion process.

Finally, the body forces and surface tractions density satisfy

$$
\boldsymbol{f}_{0} \in L^{\infty}(0, T ; H), \quad \boldsymbol{f}_{2} \in L^{\infty}\left(0, T ; L^{2}\left(\Gamma_{2}\right)^{d}\right)
$$

and the initial bonding field is such that

$$
\beta_{0} \in L^{\infty}\left(\Gamma_{3}\right), \quad 0 \leq \beta_{0}(\boldsymbol{x}) \leq 1 \text { a.e. } \boldsymbol{x} \in \Gamma_{3} .
$$

Next, we define the function $f:[0, T] \rightarrow V$ by the equality

$$
\begin{aligned}
&(\boldsymbol{f}(t), \boldsymbol{v})_{V}=\int_{\Omega} \boldsymbol{f}_{0}(t) \cdot \boldsymbol{v} d x+\int_{\Gamma_{2}} \boldsymbol{f}_{2}(t) \cdot \boldsymbol{v} d a \quad \forall \boldsymbol{v} \in V, \\
& \text { a.e. } t \in(0, T),
\end{aligned}
$$

and let $j: L^{\infty}\left(\Gamma_{3}\right) \times V \times V \rightarrow \mathbb{R}$ be the contact functional

$$
\begin{aligned}
j(\beta, \boldsymbol{u}, \boldsymbol{v}) & =\int_{\Gamma_{3}} p_{\nu}\left(u_{\nu}\right) v_{\nu} d a-\int_{\Gamma_{3}} \gamma_{\nu} \beta^{2}\left(-R_{L}\left(u_{\nu}\right)\right)_{+} v_{\nu} d a \\
& +\int_{\Gamma_{3}} \boldsymbol{p}_{\tau}\left(\beta, \boldsymbol{u}_{\tau}\right) \cdot \boldsymbol{v}_{\tau} d a \quad \forall \beta \in L^{\infty}\left(\Gamma_{3}\right), \forall \boldsymbol{u}, \boldsymbol{v} \in V .
\end{aligned}
$$

Keeping in mind (4.3), (4.4) and (4.6), it follows that the integrals in (4.8), (4.9) are well defined and, moreover,

$$
\boldsymbol{f} \in L^{\infty}(0, T ; V) .
$$

We now assume that $\boldsymbol{u}, \boldsymbol{\sigma}, \beta$ are regular functions which satisfy (3.13)(3.20) and let $\boldsymbol{v} \in V, t \in[0, T]$. Using (2.1) and (3.14) we have

$$
(\boldsymbol{\sigma}(t), \boldsymbol{\varepsilon}(\boldsymbol{v}))_{\mathcal{H}}=\int_{\Omega} \boldsymbol{f}_{0}(t) \cdot \boldsymbol{v} d x+\int_{\Gamma} \boldsymbol{\sigma}(t) \boldsymbol{\nu} \cdot \boldsymbol{v} d a,
$$

and by (3.15), (3.16) and (4.8) we find

$$
(\boldsymbol{\sigma}(t), \boldsymbol{\varepsilon}(\boldsymbol{v}))_{\mathcal{H}}=(\boldsymbol{f}(t), \boldsymbol{v})_{V}+\int_{\Gamma_{3}} \boldsymbol{\sigma}(t) \boldsymbol{\nu} \cdot \boldsymbol{v} d a .
$$

Using now (3.17), (3.18), (4.9) and (4.11) yields

$$
(\boldsymbol{\sigma}(t), \boldsymbol{\varepsilon}(\boldsymbol{v}))_{\mathcal{H}}+j(\beta(t), \boldsymbol{u}(t), \boldsymbol{v})=(\boldsymbol{f}(t), \boldsymbol{v})_{V} .
$$

To conclude, from (3.13), (3.19), (3.20) and (4.12) we obtain the following variational formulation of the problem $P$.

Problem $P_{V}$. Find a displacement field $\boldsymbol{u}:[0, T] \rightarrow V$, a stress field $\boldsymbol{\sigma}:$ $[0, T] \rightarrow \mathcal{H}_{1}$, and a bonding field $\beta:[0, T] \rightarrow L^{\infty}\left(\Gamma_{3}\right)$ such that

$$
\begin{gathered}
\boldsymbol{\sigma}(t)=\mathcal{E}(\boldsymbol{\varepsilon}(\boldsymbol{u}(t))) \quad \text { a.e. } t \in(0, T), \\
\dot{\beta}(t)=H_{a d}\left(\beta(t), R_{L}(|\boldsymbol{u}(t)|)\right), \quad \text { a.e. } t \in(0, T),
\end{gathered}
$$




$$
\begin{gathered}
(\boldsymbol{\sigma}(t), \boldsymbol{\varepsilon}(\boldsymbol{v}))_{\mathcal{H}}+j(\beta(t), \boldsymbol{u}(t), \boldsymbol{v})=(\boldsymbol{f}(t), \boldsymbol{v})_{V} \quad \forall \boldsymbol{v} \in V, \\
\text { a.e. } t \in(0, T), \\
\beta(0)=\beta_{0} .
\end{gathered}
$$

The existence of the unique solution of Problem $P_{V}$ is stated now and the proof is provided in the next section.

Theorem 4.1. Assume that (4.2)-(4.7) hold and

$$
\frac{c_{0}^{2}\left(L_{\nu}+L_{\tau}+\gamma_{\nu}\right)}{m_{\mathcal{E}}}<\frac{1}{\sqrt{2 e}} .
$$

Then there exists a unique solution $\{\boldsymbol{u}, \boldsymbol{\sigma}, \beta\}$ of problem $P_{V}$. Moreover, the solution satisfies

$$
\begin{gathered}
\boldsymbol{u} \in L^{\infty}(0, T ; V), \\
\boldsymbol{\sigma} \in L^{\infty}\left(0, T ; \mathcal{H}_{1}\right), \\
\beta \in W^{1, \infty}\left(0, T ; L^{\infty}\left(\Gamma_{3}\right)\right), \\
0 \leq \beta(t) \leq 1 \quad \forall t \in[0, T] \text {, a.e. on } \Gamma_{3} .
\end{gathered}
$$

Theorem 4.1 states the well posedness of the variational problem $P_{V}$. By this theorem we conclude that, under the assumptions (4.2)-(4.7) and if moreover the smallness assumption (4.17) holds, the mechanical problem $P$ has a unique weak solution with regularity (4.18)-(4.20).

Note that the result in Theorem 4.1 is obtained by restricting the size of the coefficients involved in the constitutive law and in the contact conditions. Whether it is a limitation of the mathematical approach, which is based on a fixed point argument, or intrinsic feature of the elastic contact models with adhesion is an open question. There seems to be some numerical and theoretical evidence that the difficulties are related to the intrinsic structure of the problem. On the other hand, the existence and uniqueness of the solution for the corresponding adhesive contact with viscoelastic materials have been established without any size restrictions, see [11] for details.

\section{Proof}

The proof of Theorem 4.1 is carried out in several steps that we present in what follows. Everywhere below we assume that (4.2)-(4.7) hold. We use Riesz's representation theorem to define the operator $E: V \rightarrow V$ by

$$
(E \boldsymbol{u}, \boldsymbol{v})_{V}=(\mathcal{E}(\boldsymbol{\varepsilon}(\boldsymbol{u})), \boldsymbol{\varepsilon}(\boldsymbol{v}))_{\mathcal{H}} \quad \forall \boldsymbol{u}, \boldsymbol{v} \in V .
$$


It follows from (4.2) that $E$ is a strongly monotone Lipschitz continuous operator. Therefore $E$ is invertible and, moreover, its inverse $E^{-1}: V \rightarrow V$ satisfies

$$
\left\|E^{-1}\left(\boldsymbol{w}_{1}\right)-E^{-1}\left(\boldsymbol{w}_{2}\right)\right\|_{V} \leq \frac{1}{m_{\mathcal{E}}}\left\|\boldsymbol{w}_{1}-\boldsymbol{w}_{2}\right\|_{V} \quad \forall \boldsymbol{w}_{1}, \boldsymbol{w}_{2} \in V .
$$

Let $\boldsymbol{\eta}$ be an arbitrary element of the space $L^{\infty}(0, T ; V)$ and denote

$$
\boldsymbol{u}^{\eta}(t)=E^{-1}(\boldsymbol{f}(t)-\boldsymbol{\eta}(t)) \quad \text { a.e. } t \in(0, T) .
$$

It follows from (4.10) that

$$
\boldsymbol{u}^{\eta} \in L^{\infty}(0, T ; V)
$$

and, moreover, (5.1) and (5.3) imply that

$$
\begin{aligned}
\left(\mathcal{E}\left(\varepsilon\left(\boldsymbol{u}^{\eta}(t)\right)\right), \boldsymbol{\varepsilon}(\boldsymbol{v})\right)_{\mathcal{H}}+(\boldsymbol{\eta}(t), \boldsymbol{v})_{V}=(\boldsymbol{f}(t), \boldsymbol{v})_{V} \quad \forall \boldsymbol{v} \in V, & \text { a.e. } t \in(0, T) .
\end{aligned}
$$

In the first step we consider the following evolutionary problem.

Problem $P_{V}^{\eta}$. Find a bonding field $\beta_{\eta}:[0, T] \rightarrow L^{\infty}\left(\Gamma_{3}\right)$ such that

$$
\begin{gathered}
\dot{\beta}_{\eta}(t)=H_{a d}\left(\beta_{\eta}(t), R_{L}\left(\left|\boldsymbol{u}^{\eta}(t)\right|\right)\right) \quad \text { a.e. } t \in(0, T), \\
\beta_{\eta}(0)=\beta_{0} .
\end{gathered}
$$

We have the following result.

Lemma 5.1. There exists a unique solution $\beta_{\eta}$ of problem $P_{V}^{\eta}$ and it satisfies (4.20). Moreover,

$$
0 \leq \beta_{\eta}(t) \leq 1 \quad \forall t \in[0, T], \text { a.e. on } \Gamma_{3} .
$$

Proof. For the sake of simplicity we suppress the dependence of various functions on $\boldsymbol{x} \in \Gamma_{3}$. Notice that the equalities and inequalities below are valid a.e. $\boldsymbol{x} \in \Gamma_{3}$. We consider the mapping $F:(0, T) \times L^{\infty}\left(\Gamma_{3}\right) \rightarrow L^{\infty}\left(\Gamma_{3}\right)$ defined by

$$
F(t, \beta)=H_{a d}\left(\beta, R_{L}\left(\left|\boldsymbol{u}^{\eta}(t)\right|\right)\right) \quad \text { a.e. } t \in(0, T), \forall \beta \in L^{\infty}\left(\Gamma_{3}\right) .
$$

Clearly, the mapping is well defined since if $\beta \in L^{\infty}\left(\Gamma_{3}\right)$ and $r: \Gamma_{3} \rightarrow$ $\mathbb{R}$ is a measurable function then conditions (4.5) imply that $\boldsymbol{x} \mapsto$ $H_{a d}\left(\boldsymbol{x}, \beta(\boldsymbol{x}), R_{L} r(\boldsymbol{x})\right) \in L^{\infty}\left(\Gamma_{3}\right)$. Moreover, it is easy to check that $F$ is Lipschitz continuous with respect to the second variable, uniformly in time; also, for all $\beta \in L^{\infty}\left(\Gamma_{3}\right), t \mapsto F(t, \beta)$ belongs to $L^{\infty}\left(0, T ; L^{\infty}\left(\Gamma_{3}\right)\right)$. Thus, the existence of a unique function $\beta_{\eta}$ which satisfies (5.6)-(5.7) follows from a version of the Cauchy-Lipschitz theorem.

To check (5.8) we suppose that $\beta_{\eta}\left(t_{0}\right)<0$ for some $t_{0} \in[0, T]$. By assumption (4.7) we have $0 \leq \beta_{\eta}(0) \leq 1$ and therefore $t_{0}>0$; moreover, 
since the mapping $t \mapsto \beta(t):[0, T] \rightarrow \mathbb{R}$ is continuous, we can find $t_{1} \in\left[0, t_{0}\right)$ such that $\beta_{\eta}\left(t_{1}\right)=0$. Now, let $t_{2}=\sup \left\{t \in\left[t_{1}, t_{0}\right] \mid \beta_{\eta}(t)=0\right\}$; then $t_{2}<t_{0}, \beta_{\eta}\left(t_{2}\right)=0$ and $\beta_{\eta}(t)<0$ for $t \in\left(t_{2}, t_{0}\right]$. Assumption (4.5)(f) and equation (5.6) imply that $\dot{\beta}_{\eta}(t) \geq 0$ for $t \in\left(t_{2}, t_{0}\right]$ and therefore $\beta_{\eta}\left(t_{0}\right) \geq$ $\beta_{\eta}\left(t_{2}\right)=0$, which is a contradiction. We conclude that $\beta_{\eta}(t) \geq 0$ for all $t \in[0, T]$. A similar argument shows that $\beta_{\eta}(t) \leq 1$ for all $t \in[0, T]$.

We now study the dependence of the solution of problem $P_{V}^{\eta}$ with respect to $\boldsymbol{\eta}$.

Lemma 5.2. Let $\boldsymbol{\eta}_{i} \in L^{\infty}(0, T ; V)$ and let $\beta_{i}$ denote the solutions of problems $P_{V}^{\eta_{i}}, i=1,2$. Then,

$$
\begin{array}{r}
\left\|\beta_{\eta_{1}}(t)-\beta_{\eta_{2}}(t)\right\|_{L^{2}\left(\Gamma_{3}\right)}^{2} \leq \frac{L_{H_{a d}}^{2} c_{0}^{2}}{m_{\mathcal{E}}^{2}} T e^{2 T L_{H_{a d}}} \int_{0}^{t}\left\|\boldsymbol{\eta}_{1}(s)-\boldsymbol{\eta}_{2}(s)\right\|_{V}^{2} d s \\
\forall t \in[0, T] .
\end{array}
$$

Proof. Let $t \in[0, T]$. The equalities and inequalities below are valid a.e. $\boldsymbol{x} \in \Gamma_{3}$ and, as usual, we do not depict the dependence on $\boldsymbol{x}$ explicitly. Using (5.6) and (5.7) we can write

$$
\beta_{i}(t)=\beta_{0}+\int_{0}^{t} H_{a d}\left(\beta_{i}(s), R_{L}\left(\left|\boldsymbol{u}^{i}(s)\right|\right) d s, \quad i=1,2\right.
$$

where $\boldsymbol{u}^{i}=\boldsymbol{u}^{\eta_{i}}$. Using now the properties (4.5) of the adhesion rate function $H_{a d}$ and the definition (3.5) of the truncation function $R_{L}$, we obtain

$$
\left|\beta_{1}(t)-\beta_{2}(t)\right| \leq L_{H_{a d}}\left(\int_{0}^{t}\left|\beta_{1}(s)-\beta_{2}(s)\right| d s+\int_{0}^{t}\left|\boldsymbol{u}^{1}(s)-\boldsymbol{u}^{2}(s)\right| d s\right) .
$$

We apply now Gronwall's inequality to deduce that

$$
\left|\beta_{1}(t)-\beta_{2}(t)\right| \leq L_{H_{a d}} e^{T L_{H_{a d}}} \int_{0}^{t}\left|\boldsymbol{u}^{1}(s)-\boldsymbol{u}^{2}(s)\right| d s,
$$

which implies that

$$
\left|\beta_{1}(t)-\beta_{2}(t)\right|^{2} \leq L_{H_{a d}}^{2} T e^{2 T L_{H_{a d}}} \int_{0}^{t}\left|\boldsymbol{u}^{1}(s)-\boldsymbol{u}^{2}(s)\right|^{2} d s .
$$

Integrating the last inequality over $\Gamma_{3}$ we find

$$
\left\|\beta_{1}(t)-\beta_{2}(t)\right\|_{L^{2}\left(\Gamma_{3}\right)}^{2} \leq L_{H_{a d}}^{2} T e^{2 T L_{H_{a d}}} \int_{0}^{t}\left\|\boldsymbol{u}^{1}(s)-\boldsymbol{u}^{2}(s)\right\|_{L^{2}\left(\Gamma_{3}\right)^{d}}^{2} d s,
$$

and keeping in mind (4.1), (5.2) and (5.3), we obtain (5.9). 
We consider now the operator $\Lambda: L^{\infty}(0, T ; V) \rightarrow L^{\infty}(0, T ; V)$ given by

$$
(\Lambda \boldsymbol{\eta}(t), \boldsymbol{v})_{V}=j\left(\beta_{\eta}(t), \boldsymbol{u}^{\eta}(t), \boldsymbol{v}\right) \quad \forall \boldsymbol{v} \in V \text {, a.e. } t \in(0, T),
$$

where $\beta_{\eta}$ denotes the solution of problem $P_{V}^{\eta}, \boldsymbol{u}^{\eta}$ is given by (5.3) and $j$ is defined by (4.9).

We have the following result.

Lemma 5.3. Under the smallness assumption (4.17), there exists a unique element $\boldsymbol{\eta}^{*} \in L^{\infty}(0, T ; V)$ such that $\Lambda \boldsymbol{\eta}^{*}=\boldsymbol{\eta}^{*}$.

Proof. Let $\boldsymbol{\eta}_{i} \in L^{\infty}(0, T ; V), \boldsymbol{u}^{i}=\boldsymbol{u}^{\eta_{i}}$ and let $\beta_{i}$ denote the solution of problem $P_{V}^{\eta_{i}}, i=1,2$. The equalities and inequalities below are valid for all $\boldsymbol{v} \in V$, a.e. $t \in(0, T)$. Using (5.10), (4.9) and the properties of the functions $p_{\nu}$ and $\boldsymbol{p}_{\tau}$ we obtain

$$
\begin{aligned}
& \left|\left(\Lambda \boldsymbol{\eta}_{1}(t)-\Lambda \boldsymbol{\eta}_{2}(t), \boldsymbol{v}\right)_{V}\right| \leq \int_{\Gamma_{3}} L_{\nu}\left|u_{\nu}^{1}(t)-u_{\nu}^{2}(t)\right|\left|v_{\nu}\right| d a \\
& +\int_{\Gamma_{3}} \gamma_{\nu}\left|\beta_{1}^{2}(t)\left(-R_{L}\left(u_{\nu}^{1}(t)\right)\right)_{+}-\beta_{2}^{2}(t)\left(-R_{L}\left(u_{\nu}^{2}(t)\right)\right)_{+}\right|\left|v_{\nu}\right| d a \\
& +\int_{\Gamma_{3}} L_{\tau}\left(\left|\beta_{1}(t)-\beta_{2}(t)\right|+\left|\boldsymbol{u}_{\tau}^{1}(t)-\boldsymbol{u}_{\tau}^{2}(t)\right|\right)\left|\boldsymbol{v}_{\tau}\right| d a .
\end{aligned}
$$

Keeping in mind (3.5) and (5.8), after some algebra the previous inequality leads to

$$
\begin{aligned}
\left|\left(\Lambda \boldsymbol{\eta}_{1}(t)-\Lambda \boldsymbol{\eta}_{2}(t), \boldsymbol{v}\right)_{V}\right| & \leq \int_{\Gamma_{3}}\left(L_{\nu}+L_{\tau}+\gamma_{\nu}\right)\left|\boldsymbol{u}^{1}(t)-\boldsymbol{u}^{2}(t)\right||\boldsymbol{v}| d a \\
& +\int_{\Gamma_{3}}\left(L_{\tau}+2 \gamma_{\nu} L\right)\left|\beta_{1}(t)-\beta_{2}(t)\right||\boldsymbol{v}| d a
\end{aligned}
$$

which implies that

$$
\begin{aligned}
\left|\left(\Lambda \boldsymbol{\eta}_{1}(t)-\Lambda \boldsymbol{\eta}_{2}(t), \boldsymbol{v}\right)_{V}\right| & \leq\left(L_{\nu}+L_{\tau}+\gamma_{\nu}\right)\left\|\boldsymbol{u}^{1}(t)-\boldsymbol{u}^{2}(t)\right\|_{L^{2}\left(\Gamma_{3}\right)^{d}}\|\boldsymbol{v}\|_{L^{2}\left(\Gamma_{3}\right)^{d}} \\
& +\left(L_{\tau}+2 \gamma_{\nu} L\right)\left\|\beta_{1}(t)-\beta_{2}(t)\right\|_{L^{2}\left(\Gamma_{3}\right)}\|\boldsymbol{v}\|_{L^{2}\left(\Gamma_{3}\right)^{d}}
\end{aligned}
$$

We use now (4.1) and the previous inequality to obtain

$$
\begin{aligned}
\left\|\Lambda \boldsymbol{\eta}_{1}(t)-\Lambda \boldsymbol{\eta}_{2}(t)\right\|_{V} & \leq c_{0}^{2}\left(L_{\nu}+L_{\tau}+\gamma_{\nu}\right)\left\|\boldsymbol{u}^{1}(t)-\boldsymbol{u}^{2}(t)\right\|_{V} \\
& +c_{0}\left(L_{\tau}+2 \gamma_{\nu} L\right)\left\|\beta_{1}(t)-\beta_{2}(t)\right\|_{L^{2}\left(\Gamma_{3}\right)} .
\end{aligned}
$$

Using now (5.11), (5.3), (5.2) and (5.9) we deduce that

$$
\begin{aligned}
\left\|\Lambda \boldsymbol{\eta}_{1}(t)-\Lambda \boldsymbol{\eta}_{2}(t)\right\|_{V}^{2} & \leq k\left\|\boldsymbol{\eta}_{1}(t)-\boldsymbol{\eta}_{2}(t)\right\|_{V}^{2} \\
& +M \int_{0}^{t}\left\|\boldsymbol{\eta}_{1}(s)-\boldsymbol{\eta}_{2}(s)\right\|_{V}^{2} d s
\end{aligned}
$$


where

$$
\begin{aligned}
k & =\frac{2 c_{0}^{4}\left(L_{\nu}+L_{\tau}+\gamma_{\nu}\right)^{2}}{m_{\mathcal{E}}^{2}}, \\
M & =\frac{2 L_{H_{a d}}^{2} c_{0}^{4}\left(L_{\tau}+2 \gamma_{\nu} L\right)^{2} T e^{2 T L_{H_{a d}}}}{m_{\mathcal{E}}^{2}} .
\end{aligned}
$$

We introduce now the notation:

$$
\begin{gathered}
I_{0}(t)=\left\|\boldsymbol{\eta}_{1}(t)-\boldsymbol{\eta}_{2}(t)\right\|_{V}^{2}, \\
I_{1}(t)=\int_{0}^{t}\left\|\boldsymbol{\eta}_{1}(s)-\boldsymbol{\eta}_{2}(s)\right\|_{V}^{2} d s, \\
I_{j}(t)=\int_{0}^{t} \int_{0}^{s_{j-1}} \cdots \int_{0}^{s_{1}}\left\|\boldsymbol{\eta}_{1}(r)-\boldsymbol{\eta}_{2}(r)\right\|_{V}^{2} d r d s_{1} \ldots d s_{j-1} \\
\forall j \in \mathbb{N}, j \geq 2 .
\end{gathered}
$$

Notice that

$$
I_{j}(t) \leq \frac{t^{j}}{j !}\left\|\boldsymbol{\eta}_{1}-\boldsymbol{\eta}_{2}\right\|_{L^{\infty}(0, T ; V)}^{2}, \quad \forall j \in \mathbb{N} .
$$

Reiterating the inequality (5.12), by using (5.14)-(5.16) we deduce that

$$
\left\|\Lambda^{p} \boldsymbol{\eta}_{1}(t)-\Lambda^{p} \boldsymbol{\eta}_{2}(t)\right\|_{V}^{2} \leq \sum_{j=0}^{p} C_{p}^{j} k^{p-j} M^{j} I_{j}(t), \quad \forall p \in \mathbb{N}, p \geq 2
$$

and, keeping in mind (5.17), we obtain

$$
\begin{aligned}
& \left\|\Lambda^{p} \boldsymbol{\eta}_{1}(t)-\Lambda^{p} \boldsymbol{\eta}_{2}(t)\right\|_{V}^{2} \\
& \leq\left(\sum_{j=0}^{p} C_{p}^{j} k^{p-j} \frac{M^{j} T^{j}}{j !}\right)\left\|\boldsymbol{\eta}_{1}-\boldsymbol{\eta}_{2}\right\|_{L^{\infty}(0, T ; V)}^{2} .
\end{aligned}
$$

It is easy to check that

$$
\sum_{j=0}^{p} C_{p}^{j} k^{p-j} \frac{M^{j} T^{j}}{j !} \leq \frac{(k p+M T)^{p}}{p !}
$$

and therefore, (5.18) implies

$$
\left\|\Lambda^{p} \boldsymbol{\eta}_{1}-\Lambda^{p} \boldsymbol{\eta}_{2}\right\|_{L^{\infty}(0, T ; V)}^{2} \leq \frac{(k p+M T)^{p}}{p !}\left\|\boldsymbol{\eta}_{1}-\boldsymbol{\eta}_{2}\right\|_{L^{\infty}(0, T ; V)}^{2} .
$$

Assume now that (4.17) hold. It follows from (5.13) that $0<k<1 / e$ and, therefore, the series $\sum_{p=1}^{\infty} \frac{(k p+M T)^{p}}{p !}$ is convergent. Consequently,

$$
\lim _{p \rightarrow \infty} \frac{(k p+M T)^{p}}{p !}=0 .
$$


It follows from (5.19) and (5.20) that for a sufficiently large $p$ the mapping $\Lambda^{p}$ is a contraction in the Banach space $L^{\infty}(0, T ; V)$. Therefore, there exists a unique $\boldsymbol{\eta}^{*} \in L^{\infty}(0, T, V)$ such that $\Lambda^{p} \boldsymbol{\eta}^{*}=\boldsymbol{\eta}^{*}$ and, moreover, $\boldsymbol{\eta}^{*}$ is the unique fixed point of $\Lambda$.

We have now all the ingredients to prove Theorem 4.1.

Proof of Theorem 4.1.

Existence. Let $\boldsymbol{\eta}^{*} \in L^{\infty}(0, T ; V)$ be the fixed point of the operator $\Lambda$ and let $\boldsymbol{u}, \beta$ be defined by (5.3), (5.6) and (5.7) for $\boldsymbol{\eta}=\boldsymbol{\eta}^{*}$, i.e. $\boldsymbol{u}=\boldsymbol{u}^{\eta^{*}}, \beta=\beta_{\eta^{*}}$. We also denote by $\boldsymbol{\sigma}$ the function given by (4.13). Clearly, (4.13), (4.14) and (4.16) hold. Since $\Lambda \boldsymbol{\eta}^{*}=\boldsymbol{\eta}^{*}$, we deduce that

$$
\left(\Lambda \boldsymbol{\eta}^{*}(t), \boldsymbol{v}\right)_{V}=\left(\boldsymbol{\eta}^{*}(t), \boldsymbol{v}\right)_{V} \quad \forall \boldsymbol{v} \in V, \text { a.e. } t \in(0, T)
$$

and, keeping in mind (5.5) and (5.10) we deduce that (4.15) holds, too. The regularity of the solution given in (4.18) follows from (5.4), while (4.20) and property (4.21) are consequences of Lemma 5.1 Moreover, since $\boldsymbol{u} \in$ $L^{\infty}(0, T ; V)$, it follows from $(4.2)$ that $\boldsymbol{\sigma} \in L^{\infty}(0, T ; \mathcal{H})$. Choosing now $\boldsymbol{v}=\boldsymbol{\varphi}$ where $\varphi \in C_{0}^{\infty}(\Omega)^{d}$ in (4.15) and using (4.8) yields

$$
\text { Div } \boldsymbol{\sigma}(t)+\boldsymbol{f}_{0}(t)=\mathbf{0}, \quad \text { a.e. } t \in(0, T) .
$$

Now, (4.6) and (5.21) imply that Div $\boldsymbol{\sigma} \in L^{\infty}(0, T ; H)$ which in turn implies (4.19). We conclude that the triple $\{\boldsymbol{u}, \boldsymbol{\sigma}, \beta\}$ is a solution of problem $P_{V}$ and it satisfies (4.18)-(4.21).

Uniqueness. The uniqueness part is a consequence of the uniqueness of the fixed point of the operator $\Lambda$. Indeed, let $\{\boldsymbol{u}, \boldsymbol{\sigma}, \beta\}$ be a solution of problem $P_{V}$ with (4.18)-(4.20) and consider the element $\boldsymbol{\eta} \in L^{\infty}(0, T ; V)$ defined by

$$
\boldsymbol{\eta}(t)=\boldsymbol{f}(t)-E \boldsymbol{u}(t) \quad \text { a.e. } t \in(0, T) .
$$

It follows from (5.3) and (5.22) that

$$
\boldsymbol{u}=\boldsymbol{u}^{\eta}
$$

and, since Lemma 5.1 states that problem $P_{V}^{\eta}$ has a unique solution denoted $\beta_{\eta},(4.14)$ and (4.16) imply that

$$
\beta=\beta_{\eta} .
$$

We now use (5.10), (4.15), (4.13) and (5.22) to see that

$$
(\Lambda \boldsymbol{\eta}(t), \boldsymbol{v})_{V}=(\boldsymbol{\eta}(t), \boldsymbol{v})_{V} \quad \forall \boldsymbol{v} \in V, \text { a.e. } t \in(0, T),
$$

which shows that $\Lambda \boldsymbol{\eta}=\boldsymbol{\eta}$. As $\Lambda$ has a unique fixed point we conclude that

$$
\boldsymbol{\eta}=\boldsymbol{\eta}^{*} \text {. }
$$


The uniqueness part of Theorem 4.1 is now a consequence of equalities (5.23)-(5.25) and (4.13).

\section{References}

[1] Andrews, K. T., Chapman, L., Fernández, J. R., Fisackerly, M., Shillor, M., Vanerian, l., VanHouten, T., A membrane in adhesive contact, SIAM J. Appl. Math. 64 (2003), $152-169$.

[2] Andrews, K. T., Shillor, M., Dynamic adhesive contact of a membrane, Adv. Math. Sci. Appl. 13 (2003), 343-356.

[3] Chau, O., Fernández, J. R., Shillor, M., Sofonea, M., Variational and numerical analysis of a quasistatic viscoelastic contact problem with adhesion, J. Comput. Appl. Math. 159 (2003), 431-465.

[4] Chau, O., Shillor, M., Sofonea, M., Dynamic frictionless contact with adhesion, J. Appl. Math. Phys. (ZAMP) 55 (2004), 32-47.

[5] Curnier, A., Talon, C., A model of adhesion added to contact with friction, in "Contact Mechanics", JAC Martins and MDP Monteiro Marques (eds.), Kluwer, Dordrecht, 2002, 161-168.

[6] Fernández, J. R., Shillor, M., Sofonea, M., Analysis and numerical simulations of a dynamic contact problem with adhesion, Math. Comput. Modelling 37 (2003), 13171333.

[7] Frémond, M., Equilibre des structures qui adhèrent à leur support, C. R. Acad. Sci. Paris Sér. II Méc. Phys. Chim. Sci. Univers Sci. Terre 295 (1982), 913-916.

[8] Frémond, M., Adhérence des solides, J. Méc. Théor. Appl. 6 (1987), 383-407.

[9] Han, W., Kuttler, K. L., Shillor, M., Sofonea, M., Elastic beam in adhesive contact, Internat. J. Solids Structures 39 (2002), 1145-1164.

[10] Han, W., Sofonea, M., Quasistatic Contact Problems in Viscoelasticity and Viscoplasticity, Stud. Adv. Math. 30, Amer. Math. Soc., Providence, RI-International Press, Somerville, MA, 2002.

[11] Hemici, N., Awbi, B., Sofonea, M., A viscoelastic frictionless contact problem with normal compliance and adhesion, An. Univ. Bucureşti Mat. 51 (2002), 145-156.

[12] Jianu, L., Shillor, M., Sofonea, M., A viscoelastic bilateral frictionless contact problem with adhesion, Appl. Anal. 80 (2001), 233-255.

[13] Nečas, J., Hlavaček, I., Mathematical Theory of Elastic and Elastoplastic Bodies: An Introduction, Elsevier, Amsterdam, 1981.

[14] Raous, M., Cangémi, L., Cocu, M., A consistent model coupling adhesion, friction, and unilateral contact, Comput. Methods Appl. Mech. Engrg. 177 (1999), 383-399.

[15] Rojek, J., Telega, J. J., Contact problems with friction, adhesion and wear in orthopaedic biomechanics. I: General developments, J. Theoret. Appl. Mech. 39 (2001), 655-677.

[16] Rojek, J., Telega, J. J., Stupkiewicz, S., Contact problems with friction, adhesion and wear in orthopaedic biomechanics. II: Numerical implementation and application to implanted knee joints, J. Theoret. Appl. Mech. 39 (2001), 679-706.

[17] Shillor, M., Sofonea, M., Telega, J. J., Models and Variational Analysis of Quasistatic Contact, Lecture Notes in Phys. 655, Springer, Berlin-Heidelberg, 2004.

[18] Sofonea, M., Matei, A., Elastic antiplane contact problem with adhesion, J. Appl. Math. Phys. (ZAMP) 53 (2002), 962-972. 
Mircea Sofonea

LABORATOIRE DE MATHÉMATIQUES

ET De Physique Pour les Systèmes

Université de Perpignan

52 Avenue Paul Alduy

66860 Perpignan, France

E-MAIL: SOFONEA@UNIV-PERP.FR
Andaluzia MATEI

DePARTEMENT OF MATHEMATICS

University of Craiova

BD. A. I. CuzA 13

200585 Craiova

ROMANiA

E-MAIL: ANDALUZIAMATEI@K.RO 